\title{
International Regulations for Bioequivalence Approval of Locally Acting Orally Inhaled Drug Products
}

\author{
Dr. Vinod Gaikwad ${ }^{1}$, Prajakta Patil ${ }^{1}$, Atmaram Pawar ${ }^{1}$, and Kakasaheb Mahadik ${ }^{1}$ \\ ${ }^{1}$ Bharati Vidyapeeth Deemed University Poona College of Pharmacy
}

January 9, 2021

\begin{abstract}
Bioequivalence (BE) is established between the brand drug and the generic drug to allow the linking of preclinical and clinical testing conducted on the reference listed drug. Regulatory agencies around the globe have come up with the guidance for locally acting orally inhaled drug products (OIDPs) for bioequivalence approaches. The prime intent of the present article is to compare approaches of different international regulatory authorities such as Health Canada, European Medicines Agency and the US Food and Drug Administration that have published guidance related to locally acting OIDPs. Moreover, the Central Drugs Standard Control Organisation, India, has published guidelines for bioavailability and bioequivalence studies. $\mathrm{BE}$ recommendations from global regulatory agencies were based on comparison for different parameters, namely inhaler device, formulation, reference product's selection, in-vitro as well as in-vivo studies (pharmacokinetics, pharmacodynamics, and clinical studies). In the case of in-vivo studies, details about study design, dose choices, inclusion/ exclusion criteria of the subject, study period, endpoint study, and equivalence acceptance criteria were discussed in the present review article.
\end{abstract}

\section{Hosted file}

=3.Review_OIDPs.pdf available at https://authorea.com/users/219680/articles/503166international-regulations-for-bioequivalence-approval-of-locally-acting-orally-inhaleddrug-products 